\title{
Síndrome de Wallenberg asociado a una arteria vertebral derecha hipoplásica bifurcada: Primer reporte de un caso en el Perú.
}

\author{
Wallenberg syndrome associated with a bifurcated hypoplastic right vertebral artery: First case report in \\ Peru.
}

\author{
Rocío Campos-Gamarra ${ }^{1, a}$, Mayra Cerna-Rodriguez ${ }^{1, a}$, José Camones-Huerta ${ }^{1, a}$, Seungseo Choi 1,a \\ Jimmy Palacios-García ${ }^{1,2, b}$, Néstor E. Najar-Trujillo ${ }^{1,2, b}$.
}

\section{RESUMEN}

El síndrome de Wallenberg es el accidente cerebrovascular isquémico más común de la circulación posterior, causado por una obstrucción aterotrombótica en la arteria vertebral o la arteria cerebelosa posteroinferior. Esta oclusión origina una isquemia en la porción lateral del bulbo que resulta en la triada representativa del síndrome de Horner, ataxia ipsilateral a la lesión y alteraciones sensitivas. El riesgo de padecer este cuadro es mayor si se tiene variaciones anatómicas tales como una arteria vertebral hipoplásica y/o duplicada. Se presenta el caso de una mujer de 45 años admitida por hipoestesia en el lado derecho del rostro y en el hemicuerpo izquierdo, así como disfagia y vómitos. La resonancia magnética reveló una lesión isquémica en la zona bulbar lateral derecha; en la angiorresonancia se evidenció una arteria vertebral derecha hipoplásica, en tanto que la angio-TEM del cuello mostró una arteria vertebral derecha hipoplásica y bifurcada. En base a la clínica y exámenes radiológicos se confirmó el diagnóstico de Síndrome de Wallenberg, causado por isquemia en el territorio de la arteria vertebral derecha hipoplásica bifurcada.

PALABRAS CLAVE: Síndrome de Wallenberg, arteria vertebral, variación anatómica.

\section{SUMMARY}

Wallenberg syndrome is the most common ischemic stroke of the posterior circulation, caused by an atherothrombotic obstruction of the vertebral or the postero-inferior cerebellar artery. This occlusion leads to ischemia in the lateral portion of the bulb, which results in the representative triad of Horner's syndrome, ataxia on the ipsilateral side of the lesion and sensory alterations. There is a greater risk of suffering from this condition with anatomical variations such as a hypoplastic and/or duplicated vertebral artery. The case of a 45-year-old woman admitted as an emergency due to hypoesthesia on the right side of the face and on the left side of the body, as well as dysphagia and vomiting is reported. MRI studies revealed an ischemic lesion in the right-side of the bulbar area; in the angio-MRI a hypoplastic right vertebral artery was evidenced whereas the angio-TC showed a hypoplastic and duplicated right vertebral artery. On the basis of the clinical and radiological examinations, the diagnosis of Wallenberg syndrome caused by bifurcated hypoplastic right vertebral artery ischemia, was confirmed.

KEYWORDS: Wallenberg syndrome, vertebral artery, anatomic variation.

\footnotetext{
Universidad Peruana Cayetano Heredia. Lima, Perú

2 Hospital Cayetano Heredia. Lima, Perú

a Estudiante de pregrado de Medicina.

b Neurólogo.
} 
Síndrome de Wallenberg asociado a una arteria vertebral derecha hipoplásica bifurcada: Primer reporte de un caso.

\section{INTRODUCCIÓN}

El síndrome de Wallenberg, también conocido como infarto bulbar lateral o síndrome de sensibilidad alterna ocurre por una oclusión aterotrombótica de la arteria vertebral intracraneal o la arteria cerebelosa posteroinferior (PICA) (1), la cual irriga la porción inferior del cerebelo y la región lateral del bulbo raquídeo. Su causa más frecuente es la arterioesclerosis, aunque también se puede originar por disecciones arteriales y otras causas menos comunes (2).

Una arteria vertebral duplicada es muy infrecuente, solo se han reportado catorce casos con hallazgos angiográficos del doble origen de la arteria vertebral de la arteria subclavia ipsilateral, es importante porque puede causar hipoperfusión de la circulación posterior (3). En este caso, la paciente presenta una arteria vertebral con una bifurcación antes de formar la arteria basilar, esta anomalía es muy infrecuente, por lo cual no está reportado.

La arteria vertebral hipoplásica es otra variante anatómica, presente hasta en el $10 \%$ de la población clínicamente sana, y se observa con mayor frecuencia en el lado derecho. Es importante ya que el estrechamiento y la oclusión se producen a una edad más temprana en un vaso hipoplásico en comparación con el vaso normal en una enfermedad aterosclerótica (4).

Presentamos el caso de una señora de 45 años con diagnóstico clínico y radiológico de síndrome de Wallenberg originada por una arteria vertebral hipoplásica y bifurcada con la finalidad de promover en la comunidad médica la sospecha clínica de esta enfermedad facilitando la temprana identificación y el manejo temprano de la condición.

\section{Presentación del caso}

Paciente mujer de 45 años de edad, natural de Huancavelica y procedente de Lima con un tiempo de enfermedad de 48 horas, de inicio brusco y curso agudo. Los síntomas inician súbitamente con hipoestesia en hemicara derecha e hipoestesia en el resto del hemicuerpo izquierdo, además cursó también con disfagia a líquidos y posteriormente a sólidos. Acude a hospital y clínica locales, donde tuvo 5 episodios de vómitos no explosivos de contenido alimentario, pero no se llegó a un diagnóstico certero ya que se la manejó como un paciente con cuadro respiratorio y psiquiátrico. Al día siguiente, el día del ingreso al Hospital Cayetano Heredia ( $\mathrm{HCH})$, mantiene síntomas neurológicos anteriormente descritos y se añade leve debilidad muscular en ambos miembros inferiores al intentar incorporarse desde el reposo. Con respecto a antecedentes personales y familiares, la paciente negó enfermedades de importancia y uso de métodos anticonceptivos.

Al examen físico en el momento del ingreso, las funciones vitales eran normales, la paciente lucía en regular estado general, estaba alerta, orientada en tiempo y espacio. El examen físico general estaba conservado, excepto el neurológico en el que se halla lo siguiente:

- Evaluación de funciones mentales superiores: conservada

- Pares craneales afectados: PC V: hipoestesia hemicara derecha; VII: parálisis facial derecha con sonrisa asimétrica; IX y X: ausencia de reflejo nauseoso derecho.

- Sistema motor: tono y fuerza muscular conservados tanto en miembros superiores como inferiores.

- Sistema de coordinación: marcha con amplitud de la base de sustentación del lado afectado e inestabilidad para girar por sí sola. Prueba de Romberg positiva

- Reflejos osteotendinosos conservados.Signos de Babinski y Hoffman negativos.

- Sensibilidad: Propioceptiva, Palestesia, Epicrítica, Termoalgésica disminuida en hemicuerpo izquierdo

- Otros exámenes: no presentó signos de frontalización ni signos meníngeos

Con respecto a los exámenes de laboratorio, en el

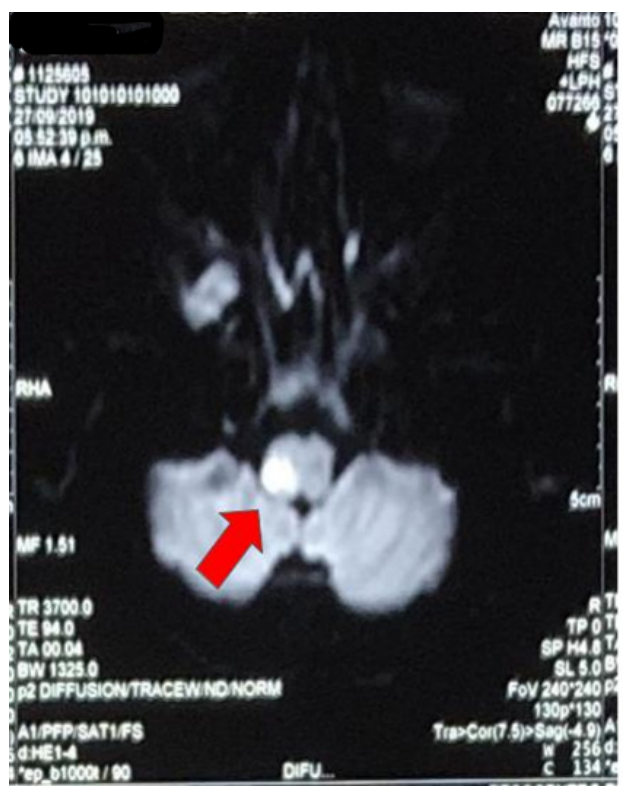

Figura 1. Resonancia magnética muestra una lesión única hiperintensa derecha. 
hemograma que incluye hemoglobina, plaquetas, urea, creatinina, perfil hepático y de coagulación estuvieron en rangos normales. En relación a los estudios de imágenes, se tomó una resonancia magnética $(25 / 09)$ (figura 1 y figura 2) muestra una lesión única hiperintensa derecha localizada en el bulbo raquídeo.

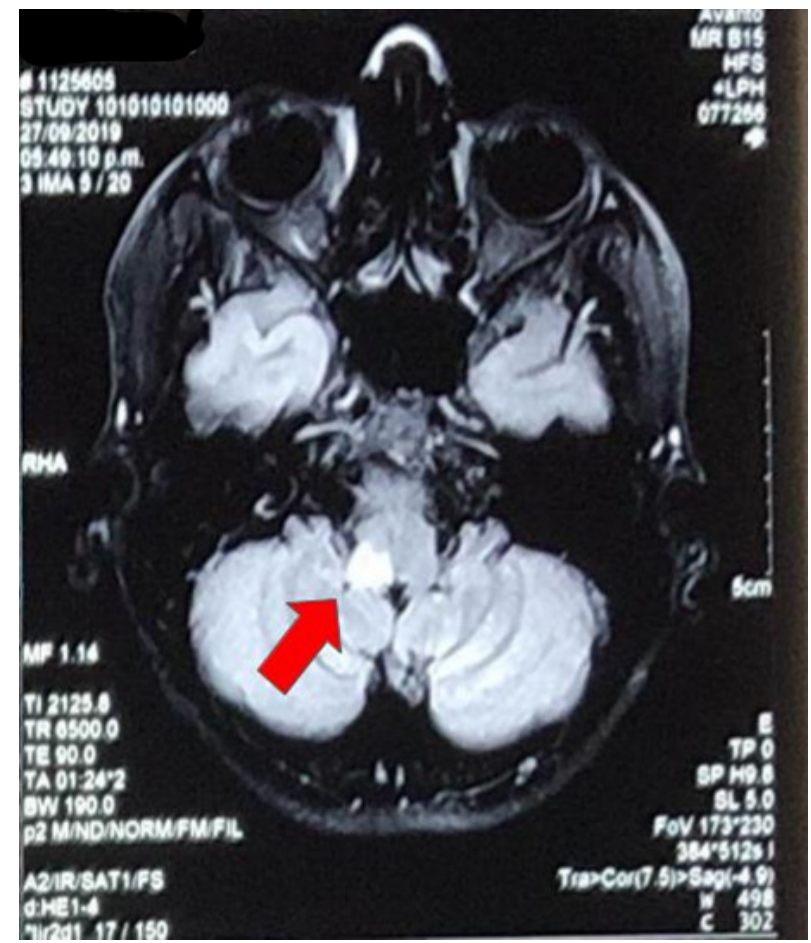

Figura 2. Resonancia magnética muestra una lesión única hiperintensa derecha.

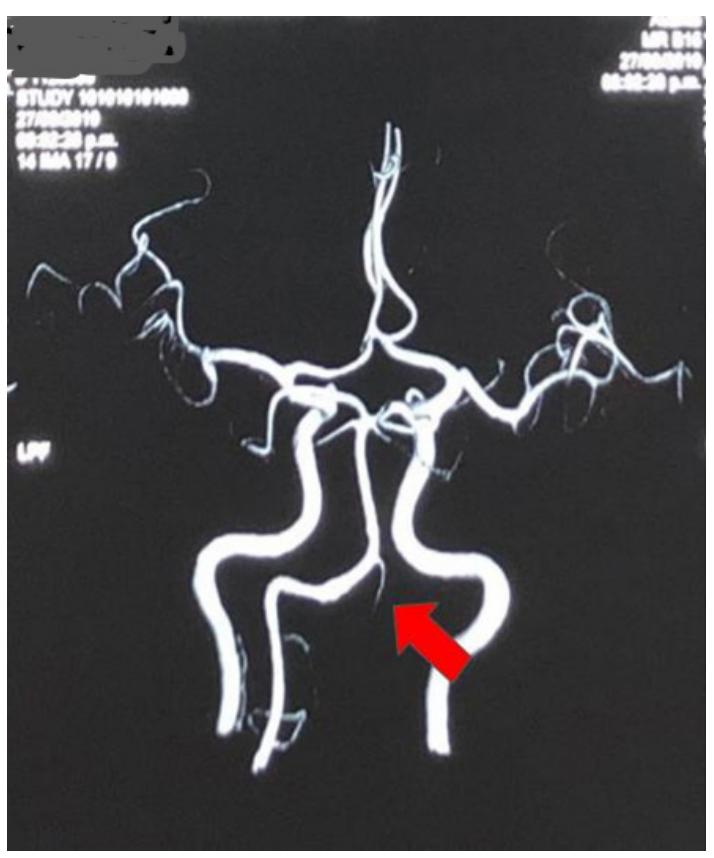

Figura 3. Angioresonancia evidencia ausencia de continuidad en la arteria vertebral derecha.
Luego se tomó una angioresonancia (27/09) (figura 3 ) en la que se evidencia ausencia de continuidad la arteria vertebral derecha, por lo que se decidió ampliar estudio a AngioTEM.

Finalmente, durante su hospitalización, se le realiza otra angioTEM de vasos supraaórticos (01/10) (figura 4) con la que se confirma una hipoplasia de la arteria vertebral derecha con bifurcación distal cerca de su unión con la arteria basilar. No se realizaron otros estudios etiológicos adicionales.

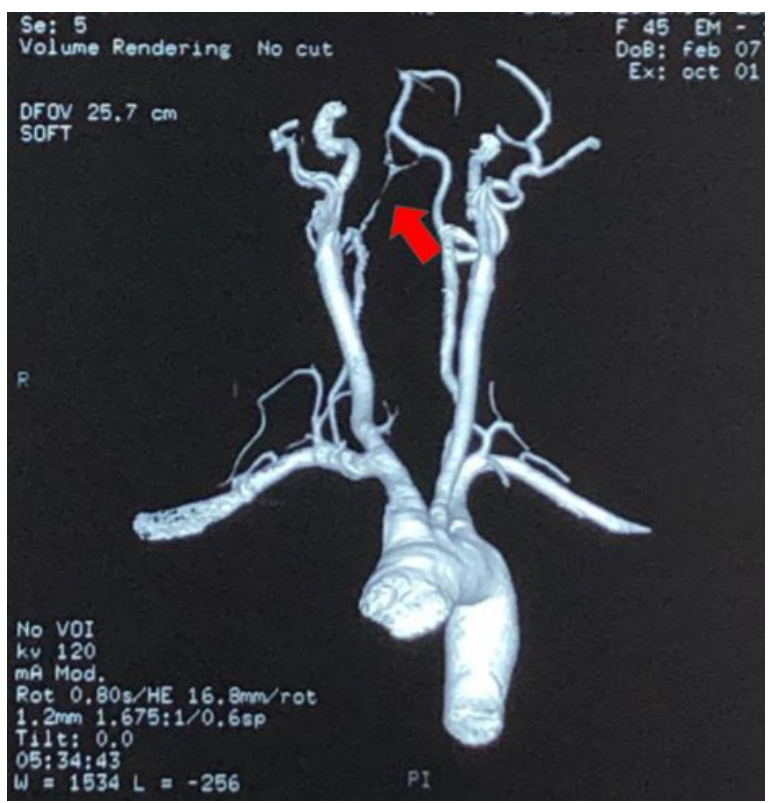

Figura 4. AngioTEM de vasos supraaórticos confirma una hipoplasia de la arteria vertebral derecha.

\section{DISCUSIÓN}

El síndrome de Wallenberg o síndrome bulbar lateral, llamado así por la primera persona que lo describió en 1895, se ocasiona por la oclusión aterotrombótica del segmento V4 de la arteria vertebral o de la arteria cerebelosa posteroinferior, la cual irriga en su segmento proximal la cara lateral del bulbo raquídeo, y con sus ramas distales la cara inferior del cerebelo.

Existen factores de riesgo para el desarrollo de este síndrome tales como hipertensión arterial, tabaquismo, Diabetes Mellitus tipo II, hiperlipidemia y cardiopatías. Es más común en hombres que en mujeres, en una proporción de 3:1 (5).

La obstrucción de estas arterias causan una variedad de síntomas debido a las estructuras afectadas. La paciente mencionada presenta una lesión en la arteria 
Síndrome de Wallenberg asociado a una arteria vertebral derecha hipoplásica bifurcada: Primer reporte de un caso.

vertebral derecha. El daño en el tracto espinotalámico causa una pérdida de sensibilidad al dolor y temperatura en la región contralateral a la lesión, en el caso de esta paciente ella tiene hipoestesia en el lado izquierdo del cuerpo. Asimismo, el daño en el tracto trigéminotalámico causa una pérdida de sensibilidad al dolor y temperatura en la región ipsilateral a la lesión en la cara, en nuestro caso la paciente presenta hipoestesia en el lado derecho de la cara. El daño en el núcleo ambiguo causa ronquera, disfagia, voz nasal, pérdida del reflejo nauseoso, parálisis ipsilateral del paladar y cuerdas vocales, presentes en esta paciente. Una afección en el núcleo vestibular ocasiona vértigo, nistagmo, náusea y vómitos, lo que explica los síntomas encontrados, y al dañarse el pedúnculo cerebelar inferior hay ataxia ipsilateral, que se traduce en la dificultad para caminar que tuvo. La tríada representativa es el síndrome de Claude-Bernard Horner, ataxia ipsilateral a la lesión, y alteraciones sensitivas como hipoestesia ipsilateral de la cara y contralateral del cuerpo(1). En el caso de la paciente se encontró ataxia e hipoestesia, pero no el síndrome de Horner.

Sin embargo, pueden haber cuadros atípicos del síndrome de Wallenberg, y el paciente tendrá alteraciones sensitivas unilaterales (contralaterales o ipsilaterales a la lesión en todo el hemicuerpo), así como cambios sensitivos bilaterales según la extensión de la lesión. Estos cuadros incompletos son más frecuentes que el cuadro típico.

Se habla de hipoplasia de la arteria vertebral cuando esta tiene un diámetro $<2-3 \mathrm{~mm}$ o cuando existe una discrepancia entre arterias de 1:1.7; y a pesar de que puede estar presente en el 10\% de personas clínicamente sanas, se la asociado a trastornos neurológicos como migraña e infarto de fosa posterior, donde la vertebral derecha suele ser la afectada $(6,7)$.

En un estudio realizado en Taiwán se encontró una incidencia de hipoplasia de la arteria vertebral e isquemia del tronco/cerebelo del 72,7\% ipsilateral; no obstante la mayoría de pacientes presentaba historia de hipertensión arterial y dislipidemia, por lo que la hipoplasia resultó ser un factor que contribuía a la isquemia, más no era un factor independiente de la causa (8). Al igual que en otros estudios donde se demuestra la frecuencia de esta hipoplasia en isquemia de circulación posterior, la principal causa fue aterosclerosis de grandes vasos (9), cabe resaltar que en estos estudios los pacientes oscilan entre 60 y 70 años.

En un estudio en China se encontró que el 10\% de personas con isquemia de fosa posterior presentaban hipoplasia de la arteria vertebral ipsilateral, donde el
$57 \%$ era por hipoplasia de la arteria vertebral derecha, a diferencia de otros estudios esta asociación no implicaba riesgos cardiovasculares como hipertensión o dislipidemias, pero sí estaba ligada al género masculino de aproximadamente 60 años (10). Otros estudios realizados en pacientes más jóvenes (40 años +-9) se encontró que la arteria vertebral hipoplásica era un factor de riesgo para la disección espontánea de arteria vertebral y para isquemia (11), como creemos que pudo haber sido el caso de nuestra paciente; otros factores que se asocian a la disección espontánea son el fumador recurrente y trauma accidental del cuello, Este último se considera como el que desencadena hipoperfusión en las arterias y genera estos eventos en personas jóvenes con hipoplasia de la arteria vertebral que no necesariamente presentan riesgo cardiovascular (12); sin embargo nuestra paciente niega traumas recientes en el cráneo, cara o cuello.

En un estudio de Reynoso y colaboradores encontraron que de un total de 240 pacientes, el polígono de Willis es clásico solo en un aproximadamente $61,3 \%$ (n:147); un 38,7\% (n:93) tiene ciertas variantes, donde, del total de variantes, se encontró que la fenestración de la arteria vertebral representó $1,1 \%$ (n:1), con una implicancia importante al momento de realizar procedimientos quirúrgicos, pero también como causa de trastornos vasculares que podrían provocar cuadros agudos como accidentes cerebro vasculares (13).

Otras variantes encontradas en la arteria vertebral, son la arteria vertebral duplicada y la arteria vertebral fenestrada. La arteria vertebral duplicada se refiere a un origen doble de la arteria en la subclavia ipsilateral, es muy rara y generalmente la rama derecha es la más afectada; de haber trombosis, suele ser leve debido a la circulación colateral, por lo que no tiene significancia clínica en la mayoría de los casos, pero es importante tenerlo en cuenta para una sospecha diagnóstica certera y especialmente si se realizara algún procedimiento o cirugía cervical anterior (14). La arteria vertebral fenestrada consiste en una separación de la arteria en dos ramas que vuelven a unirse antes de formar la arteria basilar (15), estas variantes no están presentes en la paciente.

En el caso de esta paciente se observa una bifurcación justo antes de llegar a la arteria basilar, presentando, no dos orígenes, sino dos terminaciones. La anomalía anatómica mencionada más el cuadro presente no ha sido reportada hasta la actualidad en la literatura revisada tanto de habla inglesa como española. 
Los síntomas de esta paciente fueron agudos al ingreso con manifestaciones clínicas que coincidían con la lesión posterolateral del bulbo, posteriormente se le realizó una Angio TC y resonancia magnética para confirmar el diagnóstico. En estos estudios, se demostró que la arteria vertebral derecha estaba hipoplásica y bifurcada antes de formar la arteria basilar, lo que explicaría la lesión isquémica a nivel bulbar.

El diagnóstico del síndrome de Wallenberg es clínico, se usan las imágenes para confirmar la presunción diagnóstica. El tratamiento es estándar, al paciente se le indicó una gastrostomía debido a la imposibilidad de deglutir. Si el paciente llega en las primeras 4.5 horas desde el inicio del cuadro, se le indica trombolisis IV. El pronóstico suele ser de acuerdo con el grado de afectación, con una recuperación de pocas semanas (1).

\section{CONCLUSIONES}

El diagnóstico del síndrome de Wallenberg es clínico y los estudios de imágenes sirven para aclarar la etiología. Aunque la principal causa conocida es la arterioesclerosis, hayotras patologíascomo la hipoplasia de la arteria cerebelosa posteroinferior que pueden contribuir en la ocurrencia del síndrome mencionado. Si bien no se ha encontrado tantainformación, debido a la baja incidencia de esta anomalía, acerca de la influencia de esta en eldesarrollo del síndrome, se necesita seguir investigando para poder establecer una relación certera sobre la implicancia de este hallazgo en el desarrollo del síndrome de Wallenberg.

\section{Correspondencia}

Rocío Nicole Campos Gamarra

Correo electrónico: rocio.campos.g@upch.pe

\section{Declaración de financiamiento $y$ de conflicto de} intereses:

El presente trabajo fue autofinanciado.

No existen conflictos de intereses.

\section{REFERENCIAS BIBLIOGRÁFICAS}

1. Lui F, Tadi P, Anilkumar AC. Wallenberg Syndrome. Treasure Island (FL): StatPearls Publishing; 2019. (Fecha de acceso: 13 de octubre del 2019) Disponible en: https://www.ncbi.nlm.nih.gov/books/NBK470174

2. Savitz S, Caplan L. Vertebrobasilar Disease. New England Journal of Medicine. 2005;352(25):26182626.

3. Baik J, Baek H, Shin H, Choi K. Duplication of the right vertebral artery: MRA findings and review of the literature. Springerplus. 2016;5(1):1123. doi: 10.1186/s40064-016-2807-z

4. Rajasekhar S. Duplicated and Hypoplastic V4 Segment of vertebral artery along with duplication of anterior inferior cerebellar artery: A Case Report. J Clin Diagn Res. 2017;11(8):AD03-AD05. doi: 10.7860/JCDR/2017/29157

5. Carrillo-Esper R, Rosales A, Espinoza I. Síndrome de Wallenberg. Rev Invest Med Sur Mex, 2014; 21 (3): 141-144.

6. Lacoma E, Guillén E. Arteria vertebral aberrante hipoplásica. Imagen Diagnóstica. 2017; 8(2): 64-65.

7. Trattnig S, Schwaighofer B, Hübsch P, Schwarz M, Kainberger F. Color-coded sonography of vertebral arteries. J Ultrasound Med. 1991;10:221226.

8. Chuang M, HuangC, Hu H, Yang Y. Toward a further elucidation: role of vertebral artery hypoplasia in acute ischemic stroke. European neurology. 2006; 55(4): 193-197.

9. Mitsumura H, Miyagawa S, Komatsu T, Hirai T, Kono Y, Iguchi Y. Relationship between vertebral artery hypoplasia and posterior circulation ischemia. Journal of Stroke and Cerebrovascular Diseases. 2016; 25(2): 266-269.

10. Hu Y, Li X, Liu Q, Zhang M, Wei L, Fang S, et al. Relationship between vertebral artery hypoplasia and posterior circulation stroke in Chinese patients. Neuroradiology. 2013; 55(3): 291-295

11. Zhou M, Zheng H, Gong S, Guo J, Chen N, Zhou $\mathrm{D}$, et al. Vertebral artery hypoplasia and vertebral artery dissection: a hospital-based cohort study. Neurology. 2015; 84(8): 818-824.

12. Melki E, Nasser G, Vandendries C, Adams D, Ducreux D, Denier C . Congenital vertebral duplication: a predisposing risk factor for dissection. J Neurol Sci. 2012;314(1-2):161-2. doi: 10.1016/j. jns.2011.10.030

13. Reynoso E, Meli F, Rubino P, Leconte A, Pérez M, Salas E. Variaciones del círculo arterial cerebral. valoración anatomoquirúrgica e imagenológica por ARM: Estudio de Incidencia Poblacional e Implicancia en la Ocurrencia de la Patologia Vascular. Rev. Argent Anat. 2014; 5(3):92-98.

14. Kim M. Duplicated vertebral artery : Literature review and clinical significance. J Korean Neurosurg Soc. 2018;61(1):28-34. doi: 10.3340/jkns.2017. 0202.007

15. Uchino A, Saito N, Okada Y, Kozawa E, Nishi N, Mizukoshi W, et al. Fenestrations of the intracranial vertebrobasilar system diagnosed by MR angiography. Neuroradiology. 2012; 54(5): 445-450.

Recibido: $15 / 01 / 2020$

Aceptado: 14/09/2020 